\title{
Evaluation of tympanic cavity volume by using computed tomography scan with the MATLAB and image $J$
} \author{
Abdulhakim Çoşkun ${ }^{\mathrm{e}}$ \\ ${ }^{a}$ Department of Anatomy, Erciyes University, Medical Faculty, Kayseri, Turkey \\ ${ }^{b}$ Department of Histology and Embriyology, Medical Faculty, Erciyes University, Kayseri, Turkey \\ ${ }^{c}$ Department of Biostatistic, Medical Faculty, Erciyes University, Kayseri, Turkey \\ ${ }^{d}$ Department of Biophysic, Medical Faculty, Erciyes University, Kayseri, Turkey \\ e Department of Pediatric Radiology, Medical Faculty, Erciyes University, Kayseri, Turkey
}

Niyazi Acer ${ }^{\text {* }}$, Erdoğan Unura, M. Fatih Sönmez ${ }^{\mathrm{b}}$, Gökmen Zararsız ${ }^{\mathrm{c}}$, Ayla Arslan ${ }^{\mathrm{a}}$, Mehmet Bilgen ${ }^{\mathrm{d}}$, Tolga Ertekin ${ }^{\mathrm{a}}$,

\section{ARTICLE INFO}

\section{* Correspondence to:}

Niyazi Acer

Department of Anatomy,

Faculty of Medicine,

Erciyes Universty,

Kayseri, Turkey

e-mail: acerniyazi@yahoo.com

\section{Keywords:}

Computed tomography

Image $\mathbf{J}$

MATLAB

Similarity coefficient

Tympanic cavity

\section{ABSTRACT}

The aim of this study is to calculate the tympanic cavity (TC) volume with manual tracing technique on computed tomography images of temporal bone with a software developed in house using MATLAB platform. Images with slice thickness of $0.25 \mathrm{~mm}$ were obtained from newborn cadavers and analyzed by two expert researchers independently using the software and the results were compared. The borders of TC were delineated manually and measurement of interested area was calculated on masked images. Then, the area measurements from all sections were added for the calculation of total volume. Dice similarity coefficient (DSC), Jaccard, sensitivity and specificity were used as a statistical validation metric to evaluate the performance of manual volume measurement. TC volumes were calculated as a $0.272 \pm 0.04 \mathrm{~cm}^{3}$ and $0.286 \pm 0.03 \mathrm{~cm}^{3}$ by the two researchers with MATLAB, $0.287 \pm 0.06 \mathrm{~cm}^{3}$ with Image J. DSC values being above to 0.7 indicated that the software allows consistent and reliable volume measurements. This software can potentially be applied to measure other anatomical volumes from radiological images.

J. Exp. Clin.Med., 2014; 31:121 